\title{
Optical Gating of Photoluminescence from Color Centers in Hexagonal Boron Nitride
}

\author{
Prince Khatri, Andrew J. Ramsay, Ralph Nicholas Edward Malein, Harold M. H. Chong, \\ and Isaac J. Luxmoore*
}

Cite This: https://dx.doi.org/10.1021/acs.nanolett.0c00751

Read Online

\section{ACCESS | Lill Metrics \& More | 回 Article Recommendations ｜（） Supporting Information}

ABSTRACT: We report on multicolor excitation experiments with color centers in hexagonal boron nitride at cryogenic temperatures. We demonstrate controllable optical switching between bright and dark states of color centers emitting around $2 \mathrm{eV}$. Resonant, or quasi-resonant, excitation of photoluminescence also pumps the color center, via a twophoton process, into a dark state, where it becomes trapped. Repumping back into the bright state has a step-like spectrum with a defectdependent threshold between 2.25 and $2.6 \mathrm{eV}$. This behavior is consistent with photoionization and charging between optically bright and dark states of the defect. Furthermore, a second zero phonon line, detuned by $+0.4 \mathrm{eV}$, is observed in absorption with orthogonal
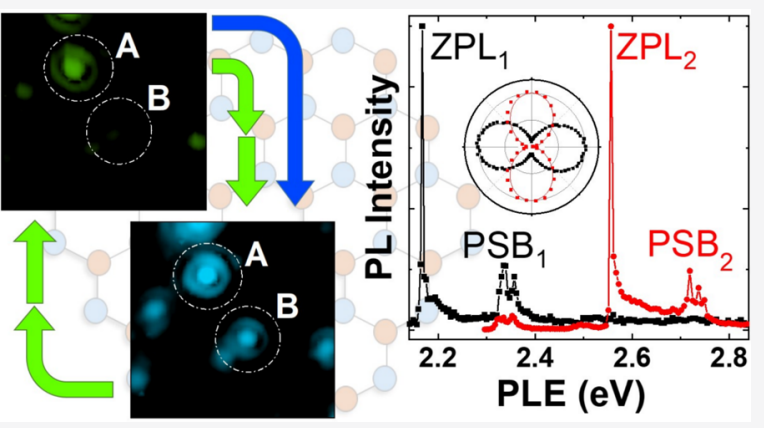
polarization to the emission, evidencing an additional energy level in the color center.

KEYWORDS: Color center, hexagonal boron-nitride, 2D materials, single photon source

$\mathrm{C}$ olor centers in wide bandgap semiconductors, most notably diamond and $\mathrm{SiC}$, with atom-like behavior and favorable spin properties, have long held potential for quantum photonic technologies, such as single photon sources and spin qubits. ${ }^{1-3}$ More recently, color centers in hexagonal boron nitride $(\mathrm{hBN})^{4-6}$ have emerged as an alternative pathway for fundamental studies, and the rapid progress reflects the wide interest and potential of this material system. These defects span a wide spectral range, from the UV to near-infrared, ${ }^{5,7-10}$ and have attractive properties for quantum optics, including narrow line width, fast radiative recombination, stable emission, and a relatively high fraction of photons emitted into the zero phonon line (ZPL). A particular benefit is the layered structure of $\mathrm{hBN}$, which allows the fabrication of twodimensional samples and integration directly with other 2D materials $^{11,12}$ and/or integrated photonic platforms. ${ }^{13}$ Furthermore, recent observations ${ }^{14,15}$ of an optically detected magnetic resonance suggests the potential to host spin qubits within a $2 \mathrm{D}$ material.

Typically, the excitation laser plays a crucial role in the optical control of quantum emitters. For example, it plays a role in stabilizing and enhancing the photoluminescence (PL) yield of color centers in diamond, ${ }^{16-20} \mathrm{SiC}^{21-23}$ and InGaAs quantum dots. ${ }^{24}$ In hBN there have been fewer studies in this area, but the choice of excitation energy has been shown to play a role in the absorption efficiency, ${ }^{25,26}$ in stabilization and enhancement of the PL emission, ${ }^{27}$ and in photochemical modification of the defect. ${ }^{28}$
In this work, we use multicolor excitation to investigate the photophysical dynamics of yellow emitting color centers in hBN. Typically green excitation is used to study such defects. The addition of a blue repump laser dramatically enhances the PL yield of certain color centers but has a weak effect on others. We investigate two defects in detail using photoluminescence, and photoluminescence excitation (PLE), spectroscopy. Through comparison with a rate equation model we find that the observed dynamics can be explained in terms of photoinduced switching between two states. The color centers are pumped into a dark state by resonant, or quasi-resonant, excitation and require a higher energy laser to repump back to the bright state. The repump spectrum is steplike with a defect specific threshold between 2.25 and $2.6 \mathrm{eV}$. This indicates a band to discrete state repump transition and points to charge state conversion as the origin of this behavior.

The sample consists of few-layer flakes of $\mathrm{hBN}$ drop-cast onto a silicon substrate coated with $5 \mathrm{~nm}$ of $\mathrm{Al}_{2} \mathrm{O}_{3}{ }^{5}$ and annealed in nitrogen at $850{ }^{\circ} \mathrm{C}$ for $8 \mathrm{~min}$. The optical experiments are performed at $5 \mathrm{~K}$ in a closed-cycle cryostat using a microphotoluminescence setup (see the Supporting

Received: February 20, 2020

Revised: $\quad$ May 8, 2020

Published: May 8, 2020 

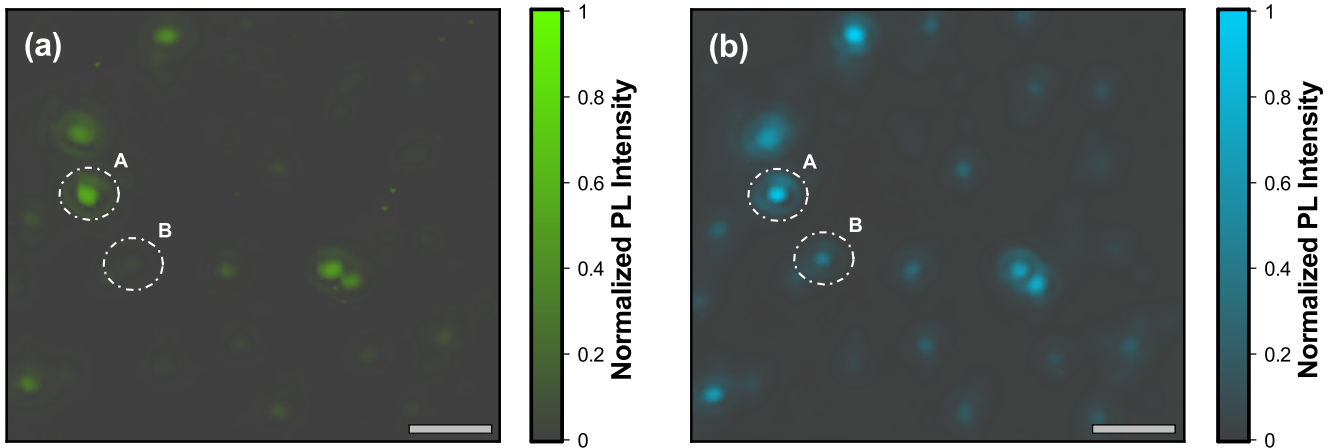

Defect-A
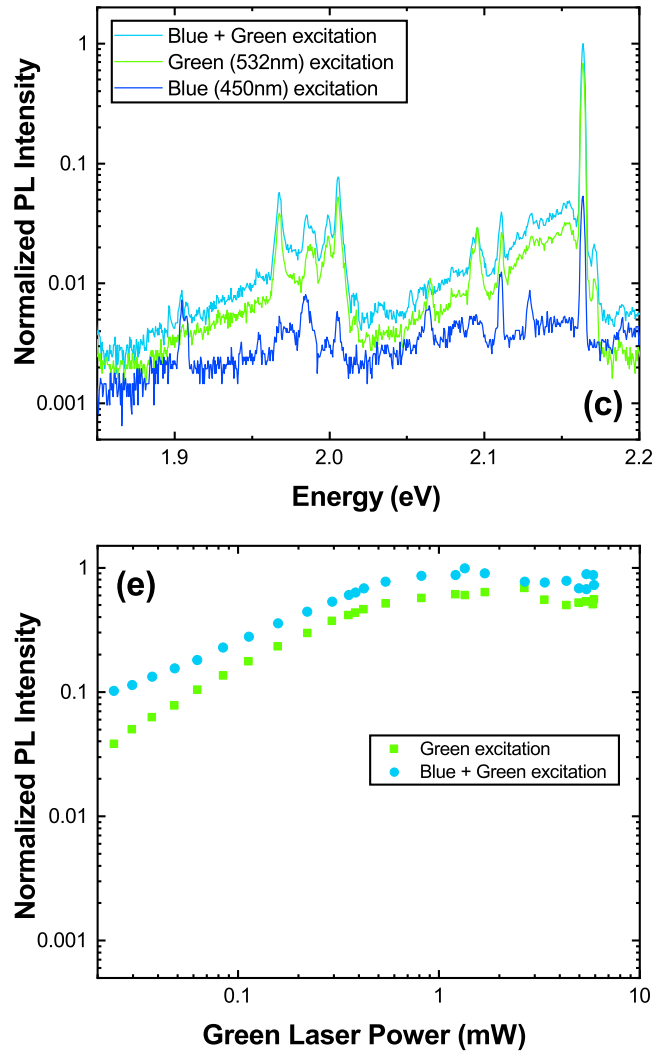

Defect-B
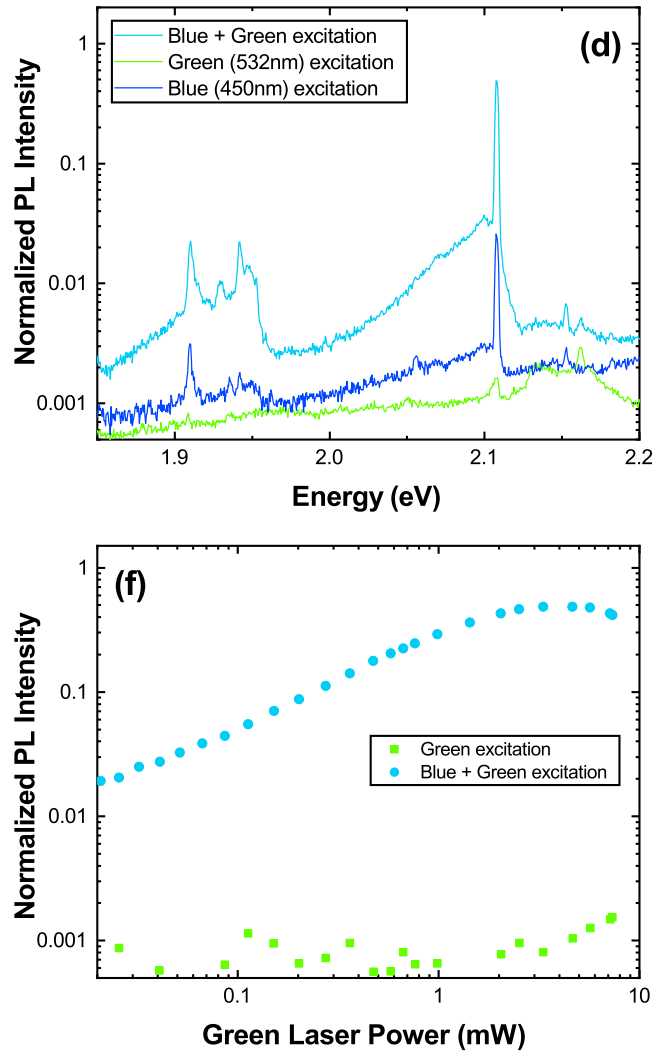

Figure 1. (a, b) Spatial maps of integrated photoluminescence emission (spectral range from 2.06 to $2.15 \mathrm{eV}$ ) under (a) green (532 nm, $2.33 \mathrm{eV}$ ) only and (b) green plus blue $(450 \mathrm{~nm}, 2.76 \mathrm{eV}$ ) excitation. The scale marker in (a) and (b) is $5 \mu \mathrm{m}$. (c, d) Photoluminescence spectra recorded under green, blue, and green plus blue excitation conditions for the two defects circled in the PL maps, (c) defect-A and (d) defect-B. The green and blue excitation powers are $\sim 700 \mu \mathrm{W}$. (e, f) Green laser power-dependent intensity of the zero phonon line PL emission for (e) defect-A and (f) defect-B, with (cyan circles) and without (green squares) the blue repump laser. In ( $c-f)$ the PL intensity is normalized to the peak counts of defect-A. In (e) and (f) the blue power is $\sim 700 \mu \mathrm{W}$.

Information (S.I.)). Three excitation lasers are used; blue and green continuous wave $(\mathrm{CW})$ excitation is provided by a 450 $\mathrm{nm}(2.76 \mathrm{eV})$ diode laser and a $532 \mathrm{~nm}(2.33 \mathrm{eV})$ diode pumped solid state (DPSS) laser, respectively, and for PLE experiments, a supercontinuum laser is filtered with an acousto-optic tunable filter (AOTF) to give a $\sim 1 \mathrm{~nm}$ bandwidth and a pulsewidth of $\sim 5$ ps. The AOTF can be driven with a superposition of radio frequencies to create a filter with multiple pass bands that can be independently controlled (wavelength and transmittance). This enables PLE measurements with simultaneous resonant excitation, as shown in Figure 4(a).

Figure 1 illustrates the enhancement of photoluminescence from color centers in hBN using multicolor excitation. Figure 1(a) shows a typical PL map, recorded with green CW excitation, which shows a number of bright spots resulting from individual, and small clusters of, color centers in hBN flakes. In Figure 1(b), the same region is mapped using coaligned green and blue CW laser beams, resulting in an approximately 2 -fold increase in the number of luminescent centers. Two representative emitters are highlighted in the PL maps and defined as defect-A and defect-B. Defect-A appears bright in both maps, whereas defect-B is dark under green excitation but bright when blue illumination is added. To verify that defects-A and -B are individual color centers, we measure the second-order autocorrelation function, $g^{(2)}(\tau)$ (see S.I.).

Further detail is shown in the PL spectra of the two defects (Figure 1(c) and (d)). Under green excitation the spectrum of 

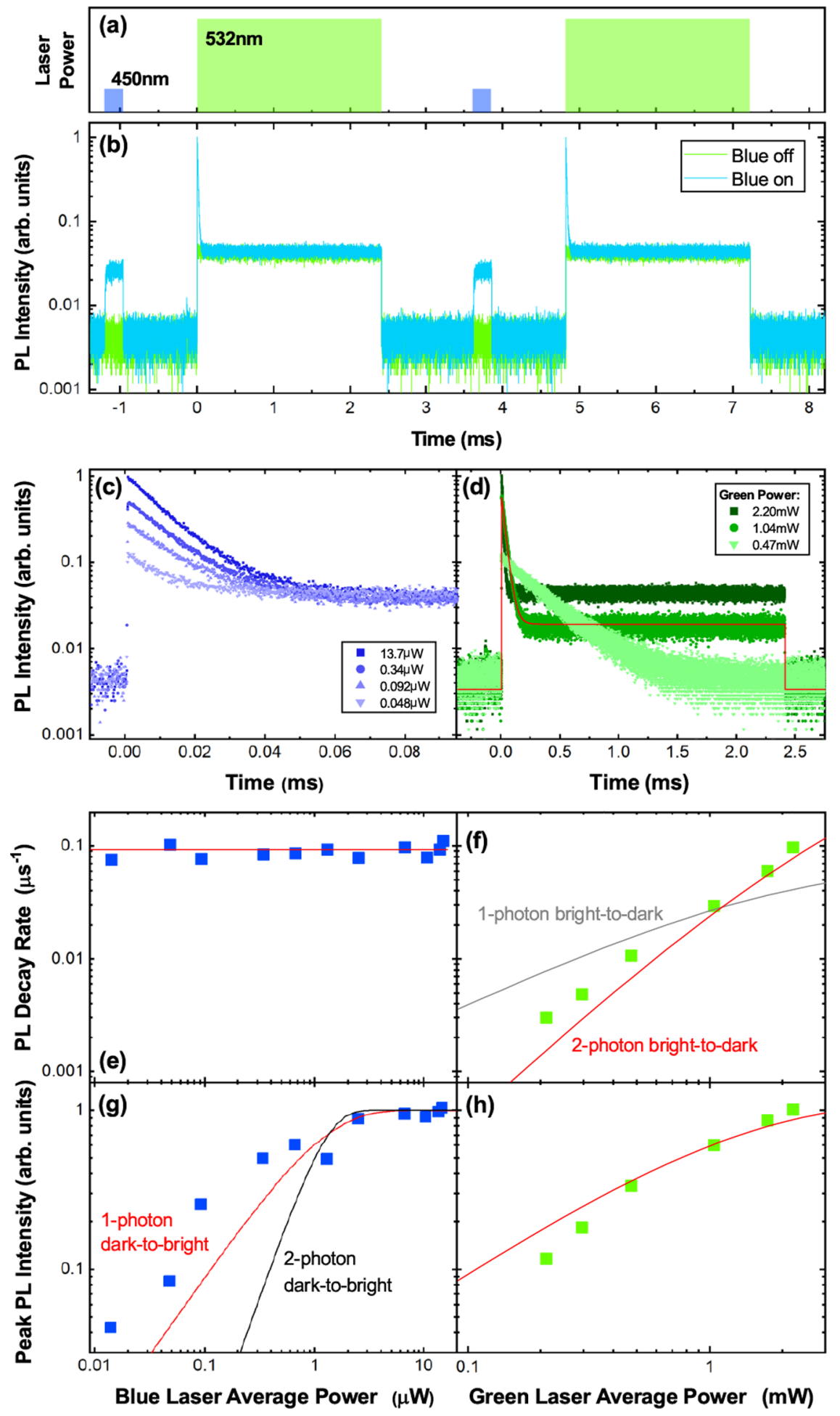

Figure 2. Time-resolved photoluminescence measurements of the zero-phonon line of defect-B. (a) Pulse-sequence used to excite photoluminescence. A blue pulse $(450 \mathrm{~nm}, 2.76 \mathrm{eV})$ of width $0.5 \mathrm{~ms}$ is followed by a green pulse $(532 \mathrm{~nm}, 2.33 \mathrm{eV})$ of $2.5 \mathrm{~ms}$, with a repetition frequency of $208 \mathrm{~Hz}$. (b) Example PL intensity time trace. Without the blue pulse, the green laser excites low intensity emission. The blue pulse weakly excites PL but also prepares the defect in the bright state. The subsequent arrival of a green pulse results in high-intensity PL, which rapidly decays, indicating the defect is pumped into the dark state. (c, d) PL decay curves resulting from the onset of a green pulse for different (c) blue pump powers (fixed green power of $2.2 \mathrm{~mW}$ ) and (d) green pump powers (fixed blue power of $10.5 \mu \mathrm{W}$ ). (e, f) Exponential decay rate as a function of (e) blue and (f) green pump power. $(\mathrm{g}, \mathrm{h})$ Peak ZPL intensity from the green laser pulse as a function of $(\mathrm{g})$ blue and (h) green laser power. The red lines in $(\mathrm{d}-\mathrm{h})$ are calculated using the rate equation model (see Figure $3(\mathrm{c})$, Table 1$)$. The gray line in (f) is a fit to the data assuming a one-photon bright-to-dark transition from $\mathrm{G}$ to $\mathrm{C}$. The black line in $(\mathrm{g})$ is a fit to the data assuming a two-photon dark-tobright transition from $\mathrm{S}$ to $\mathrm{C}$. 

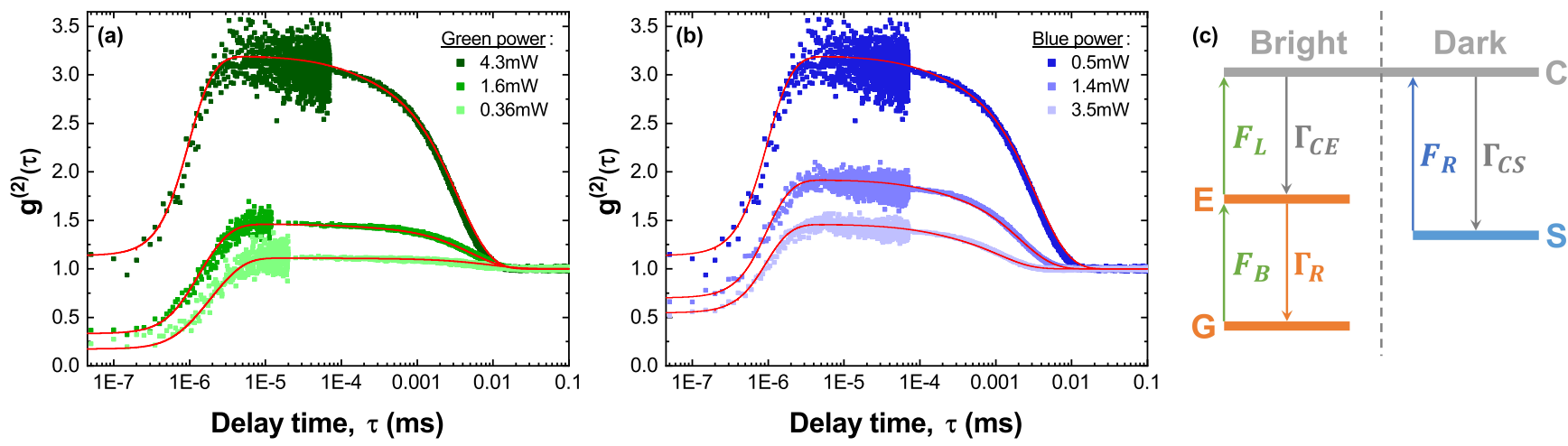

Figure 3. Second-order autocorrelation function $g^{2}(\tau)$ of defect-B with (a) fixed blue $(450 \mathrm{~nm}, 2.76 \mathrm{eV})$ power of $0.5 \mathrm{~mW}$ and varying green $(532$ $\mathrm{nm}, 2.33 \mathrm{eV}$ ) power and (b) fixed green power of $4.3 \mathrm{~mW}$ and varying blue power. The red lines show fits to the data using the model outlined in the text. (c) Rate equation model of two-photon photoionization and one-photon recharging.

defect-A, in Figure 1(c), consists of a bright ZPL at $2.167 \mathrm{eV}$, with optical and acoustic phonon sidebands (PSB) that are consistent with previous reports. ${ }^{26,29,30}$ Addition of the blue laser results in a small enhancement of the overall PL intensity. With blue excitation alone, the ZPL intensity is reduced by a factor of $\sim 20$, for the same excitation power. A comparison of the green laser power dependence of the ZPL of defect-A is made in Figure 1(e), with and without the blue laser. The presence of the blue laser increases the PL intensity at all powers of the green laser, including those beyond saturation, indicating that the blue laser is not just providing extra power but increasing the PL yield of the defect.

In the case of defect- $\mathrm{B}$, the choice of excitation conditions plays a much greater role. The PL spectrum, shown in Figure $1(\mathrm{~d})$, is similar to that of defect-A, with a single ZPL at 2.107 $\mathrm{eV}$ and similar PSBs. However, this is only the case for simultaneous excitation with blue and green lasers. With only blue excitation, the ZPL intensity is reduced by a factor of $\sim 20$, whereas for only green excitation the intensity is reduced by a factor of $\sim 400$. The power dependence (Figure 1(f)) shows that even with a green laser power up to several milliwatts the PL yield is still hundreds of times less intense without the blue laser.

To further investigate this behavior, we used time-resolved photoluminescence to study the dynamics of defect-B. Figure 2(a) illustrates the experiment, where a train of alternating blue and green pulses excite the defect, while the PL from the ZPL is directed to an avalanche photodiode. Figure 2(b) plots a typical PL time trace recorded during this experiment, with and without the blue laser. With no blue laser pulses, the photoluminescence from the defect is weak and directly follows the intensity profile of the green laser. We term this the dark state of the defect.

When the blue laser pulses are applied, weak PL is observed for the duration of the pulse. However, the blue pulse also prepares the defect in a bright state, resulting in a strong PL signal at the start of the following green pulse. The peak intensity increases linearly with blue power and saturates at a few microwatts (Figure $2(\mathrm{~g})$ ). Under green illumination, the bright state lives for tens of microseconds. The PL decays exponentially with a rate that is quadratic with green laser power, indicating that the bright-to-dark transition is mediated by a two-photon process (Figure 2(f)).

For qualitative understanding, we construct a simple rate equation model motivated by the experimental observations and illustrated in Figure 3(c). The model consists of four energy levels. $G$ and $E$ are the ground and excited levels of the bright state, respectively; $S$ represents the dark shelving state of the defect, and $C$ the conduction band or some higher energy levels of the system. $\Gamma_{\mathrm{R}}$ is the radiative decay of the bright state, and $\Gamma_{\mathrm{CS}}$ and $\Gamma_{\mathrm{CE}}$ are nonradiative transitions which link the dark and bright states. For each transition there is a corresponding optical pump. $F_{\mathrm{B}}$ is the bright state pump rate, $F_{\mathrm{L}}$ is the rate at which electrons are pumped out of the bright excited state, and $F_{\mathrm{R}}$ is the rate at which the color center is pumped out of the shelving state. The resulting system of four differential equations is solved numerically to calculate the photoluminescence, $\propto E \Gamma_{\mathrm{R}}$ (see S.I. for equations and further model details).

The model is compared to the data in Figure $2(\mathrm{e}-\mathrm{h})$ and shows good agreement with the main experimental observations. First, the rate of the dark-to-bright process, plotted in Figure 2(f), has an exponent close to 2. The model, which has a two-step (two-photon) bright-to-dark transition, reproduces the data well (red-line), whereas a fit assuming a one-photon pump directly from $\mathrm{G}$ to $\mathrm{C}$ (gray-line) does not, showing a clear preference for two-step pumping from the excited bright state. Second, Figure $2(\mathrm{~g})$ plots the peak PL at the beginning of the green pulse as a function of the average power of the blue laser, $P_{\mathrm{B}}$. At low powers, the exponent is $\sim 1$, indicating a one-photon repump process that requires an energy $>2.33 \mathrm{eV}$ (green) but $<2.76 \mathrm{eV}$ (blue). Fits assuming a one-photon $\left(F_{\mathrm{R}}\right.$ $\propto P_{\mathrm{B}}$, red-line $)$ and two-photon $\left(F_{\mathrm{R}} \propto P_{\mathrm{B}}^{2}\right.$, black-line $)$ pump from $S$ to $C$ are compared to data and verify that the onephoton model performs best (see S.I. for further discussion). Similar dynamics have been observed for germanium vacancies in diamond, where resonant excitation results in a two-step pumping of the system to a dark state, while a laser of higher energy recovers the system to a bright state with a one-photon process. $^{20}$

To further verify this model, measurements are made of $g^{(2)}(\tau)$ of defect-B for different blue and green excitation powers. In Figure $3(\mathrm{a}), g^{(2)}(\tau)$ is plotted for fixed blue power of $0.36 \mathrm{~mW}$ and three different green powers. For low green power, $g^{(2)}(\tau)$ is close to that expected for a two-level emitter. However, increasing the green power leads to pronounced bunching with an exponential decay on the order of microseconds. Similar bunching behavior has been observed previously for color centers in $\mathrm{hBN}^{5,31}$ and other wide bandgap semiconductors $^{32,33}$ and attributed to the presence of metastable dark states. In Figure 3(b), $g^{(2)}(\tau)$ is plotted for fixed green power of $4.3 \mathrm{~mW}$ and three different blue powers. 
When the blue power is low, the bunching is strong; but, increasing the blue power counteracts the tendency of the green laser to pump the defect into the dark state, and $g^{(2)}(\tau)$ close to the 2-level case is recovered. Again, similar observations have been made for the repumping of a color center in hBN but with different emission and pump energies. $^{27}$ The $g^{(2)}(\tau)$ function is calculated from the rate equation model with the Quantum Toolkit in Python package $^{34,35}$ (QuTiP) and convolved with the instrument response function of the photon counting setup (see S.I. for details and example code). By keeping the decay rates constant and varying the pump rates in proportion to the experimental powers (see Table 1), good fits to the data could be found, as plotted in Figure 3(a) and (b).

\section{Table 1. Rate Equation Model Parameters ${ }^{a}$}

\begin{tabular}{cl} 
transition & \multicolumn{1}{c}{ rate $(\mathrm{MHz})$} \\
$\Gamma_{\mathrm{R}}$ & $380 \pm 1.0$ \\
$\Gamma_{\mathrm{CE}}$ & $5.0 \pm 0.4$ \\
$\Gamma_{\mathrm{CS}}$ & $2.75 \pm 0.08$ \\
$F_{\mathrm{B}}$ & $(246.6 \pm 0.3) P_{\mathrm{G}}+(63.0 \pm 0.4) P_{\mathrm{B}}$ \\
$F_{\mathrm{L}}$ & $(6.9 \pm 0.3) \times 10^{-4} F_{\mathrm{B}}$ \\
$F_{\mathrm{R}}$ & $(0.30 \pm 0.05) P_{\mathrm{B}}$
\end{tabular}

${ }^{a_{T}}$ The radiative decay rate, $\Gamma_{\mathrm{R}}$, is extracted from a fit to time-resolved $\mathrm{PL}$ data (see S.I.). All other rates are extracted from fits to $g^{(2)}(\tau)$, as shown in Figure 3. $P_{\mathrm{G}}$ and $P_{\mathrm{B}}$ are the power (in $\mathrm{mW}$ ) of the green $(532 \mathrm{~nm})$ and blue $(450 \mathrm{~nm})$ lasers used in the experiments.

From the two-color experiments it is clear that the photon energy of the excitation laser determines if the color center is bright or dark. We therefore perform PLE spectroscopy to investigate how the optical pumping depends on photon energy. We first measure the energy threshold of the repump process for defect-B, plotted in Figure 4(b). The defect is excited by both the green $\mathrm{CW}$ laser and the tunable laser and reveals a clear energy threshold for the repump process of $\sim 2.6$ $\mathrm{eV}$. To understand why defect-A is bright under green-only illumination, a similar measurement is made. The PLE spectrum is measured with and without the addition of the blue CW laser, as plotted in Figure 4(a), while recording the intensity of the PSB emission. Without the blue laser it is not possible to resonantly excite the defect. The threshold is measured as $2.25 \mathrm{eV}$ by exciting the defect resonantly while simultaneously sweeping the repump laser, as shown in Figure 4(a). Although the ZPL energy of defect-A and -B differs by only $\sim 50 \mathrm{meV}$, the repump threshold differs by $\sim 350 \mathrm{meV}$. Nevertheless, the PLE measurements suggest that the two defects can be described by the same model but with different energy dependence of the repump rate, $F_{\mathrm{R}}$.

The simple model describes the data well, and we propose the following interpretation in terms of charge state conversion. The green laser efficiently excites the defect from the ground $(G)$ to excited state $(E)$. A second green photon can further excite the electron into the conduction band, $\mathrm{C}$. From $\mathrm{C}$, the electron can be recaptured, recharging the color center into the bright state; or, the electron is lost or trapped by another defect state, shelving the color center in a dark charge state, S. In this picture, the bright state is negatively charged with respect to the dark state (a similar process involving the valence band is also compatible with the data). A similar two-photon ionization process is responsible for photoswitching from the negative to neutral charge state of
NV-centers in diamond ${ }^{16-18}$ and out of the neutral state of the divacancy in $\mathrm{SiC}^{21,22}$ In the case of NV-centers, a two-photon process is also responsible for repumping. However, in our measurements we find that the repump process is one-photon, assuming the photon energy is sufficiently large to overcome the photocharging threshold of 2.25 and $2.6 \mathrm{eV}$, for defects $\mathrm{A}$ and $B$, respectively. The sharp, step-like, repumping spectra (Figure 4(a) and (b)) indicate a transition between a discrete energy level and a continuum of states, i.e., the conduction or valence band, thus confirming this photocharging picture. ${ }^{21-23}$ There are two possible mechanisms underlying the charging process. The first is that the measured threshold corresponds directly to the charge transfer threshold of the color center itself, and we see reasonable agreement to calculations for the boron vacancy ${ }^{36-38}(1.5$ to $2.4 \mathrm{eV})$ and $C_{\mathrm{B}} V_{\mathrm{N}}{ }^{38,39}$ (2.5 to 3.25 $\mathrm{eV}$ ) defects (see S.I.). The second explanation is that the repumping threshold represents the charging threshold of a nearby trap state, or states, resulting in free carriers that can then be captured by the color center. We note that many defect species have charging thresholds in this region. ${ }^{37,38} \mathrm{We}$ further note that in other materials, including silicon vacancies in diamond ${ }^{19}$ and $\mathrm{SiC}^{40}$ the divacancy in $4 \mathrm{H}-\mathrm{SiC},{ }^{21,23}$ and InGaAs quantum dots, ${ }^{24}$ nearby defects play a crucial role in the stabilization of the charge state of the target emitter.

The PLE measurements also reveal further information. In Figure 4(c), the PLE spectrum of defect-A is measured for excitation polarization parallel and perpendicular to the $\mathrm{ZPL}$ emission $\left(Z P L_{1}\right)$. When the polarization is parallel to $Z P L_{1}$, absorption resonances are observed in two bands detuned by $\sim 170 \mathrm{meV}$ and $\sim 340 \mathrm{meV}$, which can be attributed to the one and two optical PSBs, respectively. With orthogonal polarization the PLE spectrum is very different, with only weak absorption into the PSB but with a strong and spectrally narrow absorption resonance detuned from $Z P L_{1}$ by $400 \mathrm{meV}$, evidencing a higher energy level in the electronic structure of the color center, labeled $Z P L_{2}$ in Figure 4(c). The PLE spectrum is similar to that of $Z P L_{1}$, including a PSB detuned by $\sim 170 \mathrm{meV}$. Furthermore, the polar plot in Figure 4(c) shows that the two ZPLs are orthogonally polarized, with an $89^{\circ}$ misalignment of the absorption dipoles of $Z P L_{1}$ and $Z P L_{2}$. Although the absorption resonance is clearly observed when detecting at the energy of $Z P L_{1}$, there is no PL emission at the energy of $Z P L_{2}$ when exciting with the blue laser (S.I. Figure S3). These observations explain previous measurements where, depending on the detuning of the laser energy from the ZPL, the absorption and emission dipoles can be aligned or misaligned by up to $90^{\circ} .{ }^{6,41}$ As shown in S.I. Figure S3, when exciting with energy above (below) $Z P L_{2}$, the absorption polarization is perpendicular (parallel) to the emission of $Z P L_{1}$.

A similar PLE measurement of defect-B is presented in Figure 4(d), where low power blue excitation is used to maintain the color center in its bright state. As with defect-A, when the polarization of the tunable laser is aligned parallel to the ZPL, absorption peaks corresponding to phonon-assisted processes are observed. In this case, the relative efficiency of the two PSB is greater than for defect-A, and the three PSB is also observed. For the orthogonal polarization the absorption is considerably less efficient at all energies, but there is a weak absorption peak at $\sim 2.51 \mathrm{eV}$, labeled $Z P L_{\mathrm{B} 2}$ in Figure $4(\mathrm{~d})$. The detuning of this absorption peak is $403 \mathrm{meV}$, suggesting a similar origin as in defect-A. Similar measurements of other defects are presented in the S.I. From a survey of 16 defects, 11 

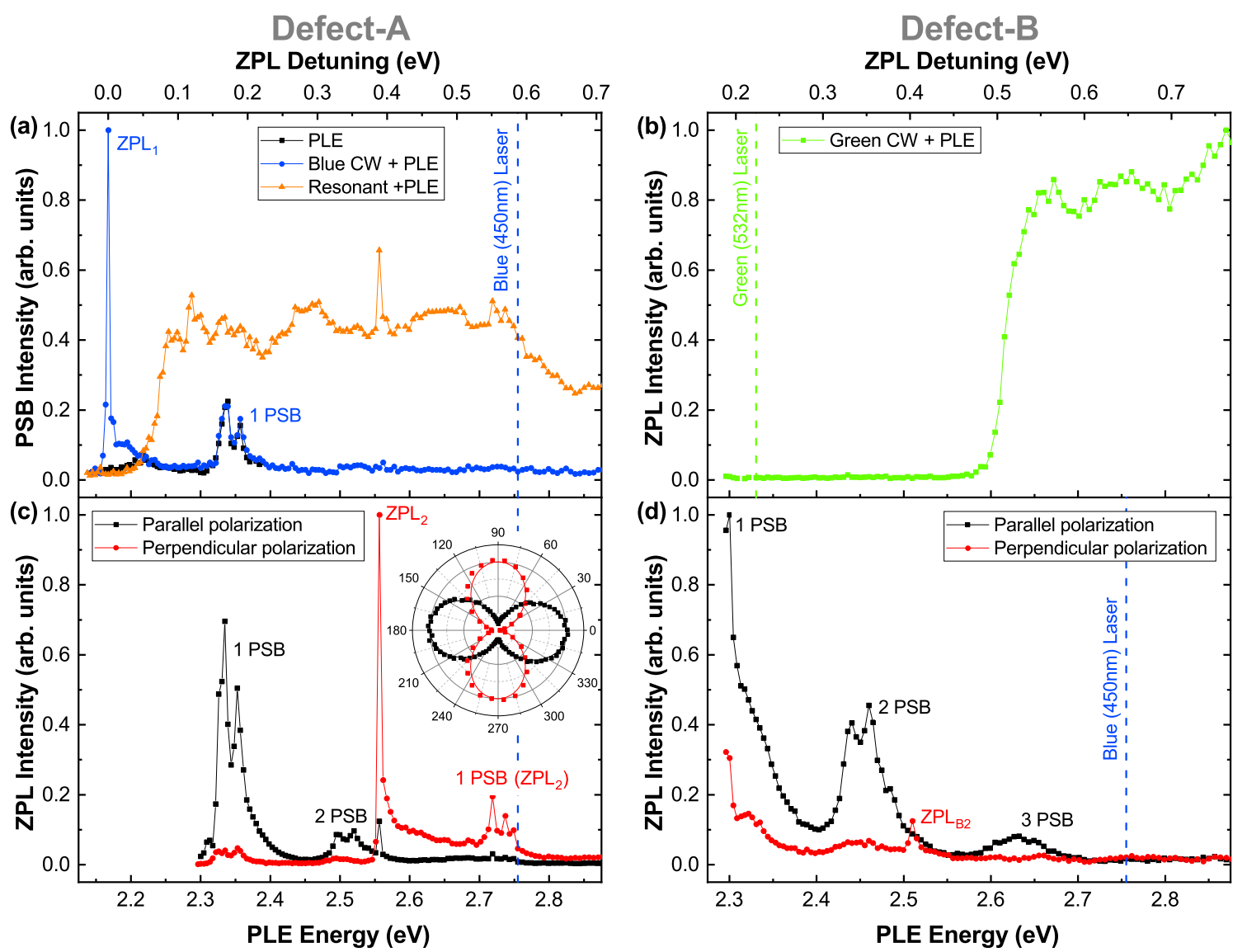

Figure 4. Photoluminescence excitation (PLE) spectroscopy. In all experiments, a tunable pulsed laser is swept across the plotted range, with the integrated intensity of the one-phonon sideband (a) or zero-phonon line (b-d) plotted for each point. (a) PLE spectra of defect-A with (blue circles) and without (black squares) blue CW laser coexcitation and with resonant coexcitation of $Z P L_{1}(2.167 \mathrm{eV}$, orange triangles). (b) PLE spectrum of defect-B with fixed green CW laser coexcitation (green squares). (c, d) PLE spectra with blue CW coexcitation for PLE laser polarization parallel (black squares) and perpendicular (red circles) to the emission from $Z P L_{1}$ for (c) defect-A and (d) defect-B. The inset to (c) shows a polar plot of the intensity of the one phonon sideband of defect- $\mathrm{A}$ as a function of the excitation polarization angle when resonant with $Z P L_{1}\left(2.167 \mathrm{eV}\right.$, black squares) and $Z P L_{2}\left(2.556 \mathrm{eV}\right.$, red circles). The black and red solid lines show $\sin ^{2} \theta$ fits to the data. In (a-d), the green and blue dashed lines indicate the energy of the green $(532 \mathrm{~nm}, 2.33 \mathrm{eV})$ and blue $(450 \mathrm{~nm}, 2.76 \mathrm{eV}) \mathrm{CW}$ lasers, respectively.

show a similar absorption peak with an average energy detuning of $0.5 \pm 0.1 \mathrm{eV}$.

Measurements of color centers in diamond again provide some clues as to the origin of $Z P L_{2}$. In the photocharging cycle of $\mathrm{NV}$ centers, resonant excitation both at the ZPL energy of $N V^{0}$ and at a higher energy level of the $N V^{-}$results in an increase in the PL yield from $\mathrm{NV}^{-} .{ }^{17}$ In $\mathrm{SiV}^{-}$a higher energy level with perpendicular polarization was identified but was not observed in PL due to selection rules of the energy levels. ${ }^{42}$ Further work is required to confirm the origin of the transition reported here, but coupled with the measured energy threshold for repumping, it provides key extra information for the identification of the defect species. When compared to theoretical predictions ${ }^{36-38,43-47}$ and other experimental work, ${ }^{48,49}$ our results suggest that the defects studied here could be boron vacancies or related to carbon impurities (see S.I. for discussion).

In conclusion, illuminating a color-center in $\mathrm{hBN}$ on, or near, resonance drives the color center into a dark state. Applying a second laser, with a photon energy exceeding a defect-specific threshold, repumps the color center back to the bright state. Phenomenologically, the dynamics can be understood in terms of photoswitching between two different charge states of the defect, as is the case for color centers in diamond and $\mathrm{SiC}^{21-23}$ Furthermore, PLE spectroscopy reveals a sharp absorption resonance, at approximately $400 \mathrm{meV}$ higher energy than the ZPL. These results are of practical use for enhancing the PL yields in both future devices and experimental studies, particularly under resonant excitation, ${ }^{50}$ and can play an important role in the identification of color centers in hBN.

\section{ASSOCIATED CONTENT}

\section{Supporting Information}

The Supporting Information is available free of charge at https://pubs.acs.org/doi/10.1021/acs.nanolett.0c00751.

Second-order autocorrelation measurements, additional photoluminescence measurements on defect-A, PLE measurements of $Z P L_{2}$ in other defects, further model details, and example QuTiP notebook (PDF)

\section{AUTHOR INFORMATION}

\section{Corresponding Author}

Isaac J. Luxmoore - College of Engineering, Mathematics and Physical Sciences, University of Exeter, Exeter EX4 4QF, United Kingdom; 이이이.org/0000-0002-2650-0842;

Email: i.j.luxmoore@exeter.ac.uk 


\section{Authors}

Prince Khatri - College of Engineering, Mathematics and Physical Sciences, University of Exeter, Exeter EX4 4QF, United Kingdom; (1) orcid.org/0000-0003-2274-5364

Andrew J. Ramsay - Hitachi Cambridge Laboratory, Hitachi Europe Limited, Cambridge CB3 OHE, United Kingdom;

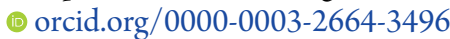

Ralph Nicholas Edward Malein - College of Engineering, Mathematics and Physical Sciences, University of Exeter, Exeter EX4 4QF, United Kingdom; (1) orcid.org/0000-0001-85023508

Harold M. H. Chong - Sustainable Electronics Technology Group, School of Electronics and Computer Science, University of Southampton, Southampton SO17 1BJ, United Kingdom; (1) orcid.org/0000-0002-7110-5761

Complete contact information is available at: https://pubs.acs.org/10.1021/acs.nanolett.0c00751

\section{Notes}

The authors declare no competing financial interest.

\section{ACKNOWLEDGMENTS}

This work was supported by the Engineering and Physical Sciences Research Council (EP/S001557/1 and EP/026656/ 1).

\section{REFERENCES}

(1) Awschalom, D. D.; Hanson, R.; Wrachtrup, J.; Zhou, B. B. Quantum technologies with optically interfaced solid-state spins. Nat. Photonics 2018, 12, 516-527.

(2) Atatüre, M.; Englund, D.; Vamivakas, N.; Lee, S.-Y.; Wrachtrup, J. Material platforms for spin-based photonic quantum technologies. Nature Reviews Materials 2018, 3, 38-51.

(3) Chen, D.; Zheludev, N.; Gao, W.-b. Building Blocks for Quantum Network Based on Group-IV Split-Vacancy Centers in Diamond. Advanced Quantum Technologies 2020, 3, 1900069.

(4) Tran, T. T.; Bray, K.; Ford, M. J.; Toth, M.; Aharonovich, I. Quantum emission from hexagonal boron nitride monolayers. Nat. Nanotechnol. 2016, 11, 37.

(5) Tran, T. T.; Elbadawi, C.; Totonjian, D.; Lobo, C. J.; Grosso, G.; Moon, H.; Englund, D. R.; Ford, M. J.; Aharonovich, I.; Toth, M. Robust Multicolor Single Photon Emission from Point Defects in Hexagonal Boron Nitride. ACS Nano 2016, 10, 7331-7338.

(6) Jungwirth, N. R.; Calderon, B.; Ji, Y.; Spencer, M. G.; Flatté, M. E.; Fuchs, G. D. Temperature Dependence of Wavelength Selectable Zero-Phonon Emission from Single Defects in Hexagonal Boron Nitride. Nano Lett. 2016, 16, 6052-6057.

(7) Vuong, T. Q. P.; Cassabois, G.; Valvin, P.; Ouerghi, A.; Chassagneux, Y.; Voisin, C.; Gil, B. Phonon-Photon Mapping in a Color Center in Hexagonal Boron Nitride. Phys. Rev. Lett. 2016, 117, No. 097402.

(8) Bourrellier, R.; Meuret, S.; Tararan, A.; Stéphan, O.; Kociak, M.; Tizei, L. H. G.; Zobelli, A. Bright UV Single Photon Emission at Point Defects in h-BN. Nano Lett. 2016, 16, 4317-4321.

(9) Li, X.; Shepard, G. D.; Cupo, A.; Camporeale, N.; Shayan, K.; Luo, Y.; Meunier, V.; Strauf, S. Nonmagnetic Quantum Emitters in Boron Nitride with Ultranarrow and Sideband-Free Emission Spectra. ACS Nano 2017, 11, 6652-6660.

(10) Shevitski, B.; Gilbert, S. M.; Chen, C. T.; Kastl, C.; Barnard, E. S.; Wong, E.; Ogletree, D. F.; Watanabe, K.; Taniguchi, T.; Zettl, A.; Aloni, S. Blue-light-emitting color centers in high-quality hexagonal boron nitride. Phys. Rev. B: Condens. Matter Mater. Phys. 2019, 100, 155419 .

(11) Geim, A. K.; Grigorieva, I. V. Van der Waals heterostructures. Nature 2013, 499, 419-425.
(12) Liu, Y.; Huang, Y.; Duan, X. Van der Waals integration before and beyond two-dimensional materials. Nature 2019, 567, 323-333.

(13) Proscia, N. V.; Jayakumar, H.; Ge, X.; Lopez-Morales, G.; Shotan, Z.; Zhou, W.; Meriles, C. A.; Menon, V. M. Scalable microcavity-coupled emitters in hexagonal boron nitride. arXiv (Optics), June 15, 2019, arXiv:1906.06546; https://arxiv.org/abs/ 1906.06546 (accessed 2020).

(14) Chejanovsky, N.; Mukherjee, A.; Kim, Y.; Denisenko, A.; Finkler, A.; Taniguchi, T.; Watanabe, K.; Dasari, D. B. R.; Smet, J. H.; Wrachtrup, J. Single spin resonance in a van der Waals embedded paramagnetic defect. arXiv (Mesoscale and Nanoscale Physics), June 13, 2019, arXiv:1906.05903; https://arxiv.org/abs/1906.05903 (accessed 2020).

(15) Gottscholl, A.; Kianinia, M.; Soltamov, V.; Orlinskii, S.; Mamin, G.; Bradac, C.; Kasper, C.; Krambrock, K.; Sperlich, A.; Toth, M.; Aharonovich, I.; Dyakonov, V. Initialization and read-out of intrinsic spin defects in a van der Waals crystal at room temperature. Nat. Mater. 2020, 19, 540.

(16) Waldherr, G.; Beck, J.; Steiner, M.; Neumann, P.; Gali, A.; Frauenheim, T.; Jelezko, F.; Wrachtrup, J. Dark States of Single Nitrogen-Vacancy Centers in Diamond Unraveled by Single Shot NMR. Phys. Rev. Lett. 2011, 106, 157601.

(17) Beha, K.; Batalov, A.; Manson, N. B.; Bratschitsch, R.; Leitenstorfer, A. Optimum Photoluminescence Excitation and Recharging Cycle of Single Nitrogen-Vacancy Centers in Ultrapure Diamond. Phys. Rev. Lett. 2012, 109, No. 097404.

(18) Aslam, N.; Waldherr, G.; Neumann, P.; Jelezko, F.; Wrachtrup, J. Photo-induced ionization dynamics of the nitrogen vacancy defect in diamond investigated by single-shot charge state detection. New J. Phys. 2013, 15, No. 013064.

(19) Dhomkar, S.; Zangara, P. R.; Henshaw, J.; Meriles, C. A. OnDemand Generation of Neutral and Negatively Charged SiliconVacancy Centers in Diamond. Phys. Rev. Lett. 2018, 120, 117401.

(20) Chen, D.; Mu, Z.; Zhou, Y.; Fröch, J. E.; Rasmit, A.; Diederichs, C.; Zheludev, N.; Aharonovich, I.; Gao, W.-b. Optical Gating of Resonance Fluorescence from a Single Germanium Vacancy Color Center in Diamond. Phys. Rev. Lett. 2019, 123, No. 033602.

(21) Wolfowicz, G.; Anderson, C. P.; Yeats, A. L.; Whiteley, S. J.; Niklas, J.; Poluektov, O. G.; Heremans, F. J.; Awschalom, D. D. Optical charge state control of spin defects in $4 \mathrm{H}-\mathrm{SiC}$. Nat. Commun. 2017, 8, 1876.

(22) Golter, D. A.; Lai, C. W. Optical switching of defect charge states in 4H-SiC. Sci. Rep. 2017, 7, 13406.

(23) Magnusson, B.; Son, N. T.; Csóré, A.; Gällström, A.; Ohshima, T.; Gali, A.; Ivanov, I. G. Excitation properties of the divacancy in $4 \mathrm{H}$ SiC. Phys. Rev. B: Condens. Matter Mater. Phys. 2018, 98, 195202.

(24) Nguyen, H. S.; Sallen, G.; Voisin, C.; Roussignol, P.; Diederichs, C.; Cassabois, G. Optically Gated Resonant Emission of Single Quantum Dots. Phys. Rev. Lett. 2012, 108, No. 057401.

(25) Schell, A. W.; Svedendahl, M.; Quidant, R. Quantum Emitters in Hexagonal Boron Nitride Have Spectrally Tunable Quantum Efficiency. Adv. Mater. 2018, 30, 1704237.

(26) Wigger, D.; Schmidt, R.; Del Pozo-Zamudio, O.; Preuß, J. A.; Tonndorf, P.; Schneider, R.; Steeger, P.; Kern, J.; Khodaei, Y.; Sperling, J.; de Vasconcellos, S. M.; Bratschitsch, R.; Kuhn, T. Phonon-assisted emission and absorption of individual color centers in hexagonal boron nitride. 2D Mater. 2019, 6, No. 035006.

(27) Kianinia, M.; Bradac, C.; Sontheimer, B.; Wang, F.; Tran, T. T.; Nguyen, M.; Kim, S.; Xu, Z.-Q.; Jin, D.; Schell, A. W.; Lobo, C. J.; Aharonovich, I.; Toth, M. All-optical control and super-resolution imaging of quantum emitters in layered materials. Nat. Commun. 2018, 9, 874.

(28) Shotan, Z.; Jayakumar, H.; Considine, C. R.; Mackoit, M.; Fedder, H.; Wrachtrup, J.; Alkauskas, A.; Doherty, M. W.; Menon, V. M.; Meriles, C. A. Photoinduced Modification of Single-Photon Emitters in Hexagonal Boron Nitride. ACS Photonics 2016, 3, 24902496. 
(29) Khatri, P.; Luxmoore, I. J.; Ramsay, A. J. Phonon sidebands of color centers in hexagonal boron nitride. Phys. Rev. B: Condens. Matter Mater. Phys. 2019, 100, 125305.

(30) Feldman, M. A.; Puretzky, A.; Lindsay, L.; Tucker, E.; Briggs, D. P.; Evans, P. G.; Haglund, R. F.; Lawrie, B. J. Phonon-induced multicolor correlations in hBN single-photon emitters. Phys. Rev. B: Condens. Matter Mater. Phys. 2019, 99, No. 020101.

(31) Sontheimer, B.; Braun, M.; Nikolay, N.; Sadzak, N.; Aharonovich, I.; Benson, O. Photodynamics of quantum emitters in hexagonal boron nitride revealed by low-temperature spectroscopy. Phys. Rev. B: Condens. Matter Mater. Phys. 2017, 96, 121202.

(32) Aharonovich, I.; Castelletto, S.; Simpson, D. A.; Greentree, A. D.; Prawer, S. Photophysics of chromium-related diamond singlephoton emitters. Phys. Rev. A: At., Mol., Opt. Phys. 2010, 81, No. 043813.

(33) Neu, E.; Agio, M.; Becher, C. Photophysics of single silicon vacancy centers in diamond: implications for single photon emission. Opt. Express 2012, 20, 19956-19971.

(34) Johansson, J.; Nation, P.; Nori, F. QuTiP: An open-source Python framework for the dynamics of open quantum systems. Comput. Phys. Commun. 2012, 183, 1760-1772.

(35) Johansson, J.; Nation, P.; Nori, F. QuTiP 2: A Python framework for the dynamics of open quantum systems. Comput. Phys. Commun. 2013, 184, 1234-1240.

(36) Abdi, M.; Chou, J.-P.; Gali, A.; Plenio, M. B. Color Centers in Hexagonal Boron Nitride Monolayers: A Group Theory and Ab Initio Analysis. ACS Photonics 2018, 5, 1967-1976.

(37) Strand, J.; Larcher, L.; Shluger, A. L. Properties of intrinsic point defects and dimers in hexagonal boron nitride. J. Phys.: Condens. Matter 2020, 32, No. 055706.

(38) Weston, L.; Wickramaratne, D.; Mackoit, M.; Alkauskas, A.; Van de Walle, C. G. Native point defects and impurities in hexagonal boron nitride. Phys. Rev. B: Condens. Matter Mater. Phys. 2018, 97, 214104.

(39) Wu, F.; Galatas, A.; Sundararaman, R.; Rocca, D.; Ping, Y. Firstprinciples engineering of charged defects for two-dimensional quantum technologies. Phys. Rev. Materials 2017, 1, No. 071001.

(40) Widmann, M.; et al. Electrical Charge State Manipulation of Single Silicon Vacancies in a Silicon Carbide Quantum Optoelectronic Device. Nano Lett. 2019, 19, 7173-7180.

(41) Jungwirth, N. R.; Fuchs, G. D. Optical Absorption and Emission Mechanisms of Single Defects in Hexagonal Boron Nitride. Phys. Rev. Lett. 2017, 119, No. 057401.

(42) Rogers, L. J.; Jahnke, K. D.; Doherty, M. W.; Dietrich, A.; McGuinness, L. P.; Müller, C.; Teraji, T.; Sumiya, H.; Isoya, J.; Manson, N. B.; Jelezko, F. Electronic structure of the negatively charged silicon-vacancy center in diamond. Phys. Rev. B: Condens. Matter Mater. Phys. 2014, 89, 235101.

(43) Turiansky, M. E.; Alkauskas, A.; Bassett, L. C.; Van de Walle, C. G. Dangling Bonds in Hexagonal Boron Nitride as Single-Photon Emitters. Phys. Rev. Lett. 2019, 123, 127401.

(44) Ivády, V.; Barcza, G.; Thiering, G.; Li, S.; Hamdi, H.; Legeza, Ö.; Chou, J.-P.; Gali, A. Ab initio theory of negatively charged boron vacancy qubit in hBN. arXiv (Mesoscale and Nanoscale Physics), October 17, 2019, arXiv:1910.07767; https://arxiv.org/abs/1910. 07767 (accessed 2020).

(45) Attaccalite, C.; Bockstedte, M.; Marini, A.; Rubio, A.; Wirtz, L. Coupling of excitons and defect states in boron-nitride nanostructures. Phys. Rev. B: Condens. Matter Mater. Phys. 2011, 83, 144115.

(46) Smart, T. J.; Wu, F.; Govoni, M.; Ping, Y. Fundamental principles for calculating charged defect ionization energies in ultrathin two-dimensional materials. Phys. Rev. Materials 2018, 2, 124002.

(47) Wang, D.; Sundararaman, R. Substrate effects on charged defects in two-dimensional materials. Phys. Rev. Materials 2019, 3, No. 083803.

(48) Feng, J.; Deschout, H.; Caneva, S.; Hofmann, S.; Lončarić, I.; Lazić, P.; Radenovic, A. Imaging of Optically Active Defects with Nanometer Resolution. Nano Lett. 2018, 18, 1739-1744.
(49) Mendelson, N.; Chugh, D.; Cheng, T. S.; Gottscholl, A.; Long, H.; Mellor, C. J.; Zettl, A.; Dyakonov, V.; Beton, P. H.; Novikov, S. V.; Jagadish, C.; Tan, H. H.; Toth, M.; Bradac, C.; Aharonovich, I. Identifying Carbon as the Source of Visible Single Photon Emission from Hexagonal Boron Nitride. arXiv (Applied Physics), April 20, 2020, arXiv:2003.00949; https://arxiv.org/abs/2003.00949 (accessed 2020).

(50) Konthasinghe, K.; Chakraborty, C.; Mathur, N.; Qiu, L.; Mukherjee, A.; Fuchs, G. D.; Vamivakas, A. N. Rabi oscillations and resonance fluorescence from a single hexagonal boron nitride quantum emitter. Optica 2019, 6, 542-548. 